\title{
Nordiques
}

35 | 2018

Tove Jansson : Par delà les genres. Pratiques

linguistiques dans le Norden du XXIe siècle : Quels

enjeux sociaux?

\section{Nordisk dialektkorpus : introduction aux recherches sur les parlers dans le Norden}

\section{Åshild Søfteland}

Traducteur : Sarah Harchaoui

\section{CpenEdition}

\section{Journals}

Édition électronique

URL : https://journals.openedition.org/nordiques/1604

ISSN : 2777-8479

\section{Éditeur :}

Association Norden, Bibliothèque de Caen la mer

\section{Édition imprimée}

Date de publication : 1 mai 2018

Pagination : $95-115$

ISBN : 979-1-0959140-1-3

ISSN : $1761-7677$

\section{Référence électronique}

Åshild Søfteland, « Nordisk dialektkorpus : introduction aux recherches sur les parlers dans le Norden », Nordiques [En ligne], 35 | 2018, mis en ligne le 02 février 2021, consulté le 06 octobre 2021. URL http://journals.openedition.org/nordiques/1604 


\section{Nordisk dialektkorpus : introduction aux recherches sur les parlers dans le Norden ${ }^{1}$}

Åshild Softeland $^{*}$

\section{RÉSUMÉ}

L'article fait découvrir le Corpus des dialectes nordiques aux étudiants en langues scandinaves. Nous décrivons d'abord comment établir un corpus d'énoncés spontanés, en y incluant des détails sur la collecte des données, leur transcription et leur étiquetage. Toutes les langues nordiques sont représentées dans le corpus, mais cet article traite surtout le norvégien. Il donne en outre des conseils basiques sur les possibilités de formuler des recherches dans le corpus, simples aussi bien qu'avancées. Nous fournissons en supplément un tutoriel qui illustre, avec commentaires, les différentes recherches mentionnées dans le texte. La dernière section évoque des cas concrets, et discute comment le corpus peut être mis en ouvre pour les rechercher et les enseigner.

\section{ABSTRACT}

The article introduces students of Scandinavian languages to the Nordic Dialect Corpus. The paper starts with a description of the process of making a corpus of spontaneous speech, with some details on data collection, transcription and tagging. All the Nordic languages are represented in the corpus, though the paper focuses primarily on Norwegian. Furthermore, the text presents basic guidelines for composing searches in the corpus, both simple and extended. A screencast is provided as supplement to the paper, showing and commenting on the examples of search listed in the text. The last sections describe and discuss new, real-life examples on how the corpus can be used for research and teaching.

* Åshild Søfteland a obtenu son doctorat en dialectologie en 2014. Elle est désormais maître de conférences à Høgskolen i Östfold (Institut supérieur d'Östfold) en Norvège.

1 Cet article décrit le Nordisk dialektkorpus (Corpus de dialectes nordiques), développé à Tekstlaboratoriet (Laboratoire de textes) à l'université d'Oslo sous la direction de $\mathrm{M}^{\mathrm{me}}$ le professeur Janne B. Johannessen. Nous avons nous-même travaillé comme assistante et boursière au Département en question, mais cet article comporte un compte rendu général fondé sur de l'information globalement disponible dans ce corpus. 
Cet article expose la façon dont les étudiants en langues nordiques peuvent utiliser un corpus d'énoncés spontanés pour mener à bien leurs propres recherches. S'appuyant sur une description de la constitution du corpus et sur ses méthodes d'utilisation, il étudie des exemples concrets d'exploitation en situation de recherche ou d'enseignement. Le Corpus des dialectes nordiques (Nordisk dialektkorpus) comprend, comme son nom l'indique, des données dialectales des langues nordiques, c'est-à-dire le norvégien, le suédois (ainsi que l'älvdalien / älvdalsk), le danois, l'islandais et le féroïen. Le terme de " dialecte " est ici défini comme un parler spontané d'une zone géographique donnée. Celui de " corpus " renvoie à des transcriptions, annotées selon leur forme orthographique, leur catégorie grammaticale et leur forme flexionnelle. Le corpus comporte des enregistrements audio, réunis dans une base de données et rendus accessibles aux études menées par des chercheurs et des étudiants en langues.

Après avoir introduit le propos par une description sur les origines et la mise en œuvre des enregistrements du Nordisk dialektkorpus, l'article explorera le traitement automatique des données, détaillera les modalités de recherches par variation dialectale et conclura par des exemples concrets d'utilisation.

\section{QU'EST-CE QUE LE NORDISK DIALEKTKORPUS?}

Nordisk dialektkorpus ${ }^{2}$ dépend du projet Scandinavian Dialect Syntax (ScanDiaSyn), administré par Tekstlaboratoriet de l'université d'Oslo. ScanDiaSyn, lié au Nordic Centre of Excellence in Micro-comparative Syntax (NORMS) de l'université de Tromsø, a réuni un réseau de linguistes de tous les pays nordiques, et rassemblé des enregistrements de dialectes collectés principalement entre 2006 et 2010. Plusieurs instituts de recherche nordiques, comme les universités d'Oslo, de Tromsø, d'Århus ou d'Islande, ainsi que des chercheurs, ont participé au travail de terrain et à la collecte de données. Une partie des enregistrements ont été faits en lien avec des ateliers de dialectologie organisés par les membres du réseau ScanDiaSyn, dans l'ensemble des pays nordiques ${ }^{3}$.

Les données récoltées sont hétérogènes et comprennent : des interviews, réalisées par des linguistes de terrain (A) ; des discours spontanés entre deux participants, le plus souvent des amis ou des connaissances (B); des questionnaires par lesquels l'informant fournit des jugements de grammaticalité (C). Les discours spontanés (types A et B) forment la base du Nordisk dialektkorpus. Les questionnaires ont été réunis dans Nordisk syntaksdatabase ${ }^{4}$, et ne seront pas traités dans

2 http://tekstlab.uio.no/nota/scandiasyn/.

3 Pour de l'information supplémentaire sur les départements et les personnes impliqués, voir www. tekstlab.uio.no/nota/scandiasyn/dialect_data_collection.html.

4 https://tekstlab.uio.no/nsd. 
cet article ${ }^{5}$ La plupart des participants norvégiens ont participé aux interviews et aux conversations, le plus souvent avec une personne originaire d'une même zone dialectale. Les entretiens paraissent plus formels et plus courts que les discours spontanés, plus longs et plus informels. Par conséquent, les discours informels ont plus d'importance dans le corpus norvégien.

\section{Les données en chiffres}

\section{Norvégien}

- 575 personnes de 165 localités;

- Collecte systématique des données ;

- Ateliers de dialectologie organisés à Fosen, Oppdal, Senja, Surnadal et en

Scandinavie intérieure ;

- Enregistrements plus anciens de Målførearkivet ${ }^{6}$.

\section{$\underline{\text { Danois }}$}

- 81 personnes de 15 localités ;

- Collecte systématique des données ;

- Atelier de dialectologie dans le Jutland de l'Ouest.

\section{$\underline{\text { Suédois }}$}

- 150 personnes de 44 localités ;

- Ateliers de dialectologie dans l'Älvdalen, la région d'Österbotten et en

Scandinavie intérieure (le corpus ne comprend pas encore [sept. 2017]

le suédois de Finlande) ;

- Enregistrements plus anciens de SweDia 20007.

\section{Islandais}

- 48 personnes de 8 localités;

- Corpus comprenant principalement des enregistrements de 2013 et de 2015.

Le travail de terrain a eu lieu dans des contextes différents, mais les données du corpus disponibles en septembre 2017 ont été recueillies en 2013 et en 2015.

\section{Féroïen}

- 20 personnes de 5 localités;

- Données récoltées lors d'un atelier organisé aux îles Féroé.

La carte $\mathrm{n}^{\circ} 1$ montre les lieux dans lesquels les enregistrements ont été faits.

5 Pour une discussion intéressante sur la complémentarité des données spontanées et les questionnaires, voir Øystein Vangsnes, Janne B. Johannessen, "Reconciling Corpus and Questionnaire Data in Microcomparative Syntax : A Case Study from North Germanic ", in Studies in Language Variation. European Perspectives III, F. Gregersen, J. K. Parrott et P. Quist (dir.), Amsterdam, John Benjamins, 2011, p. $135-140$.

6 www.uib.no/lle/73605/meir-om-målføresamlinga.

7 http://swedia.ling.gu.se/. 


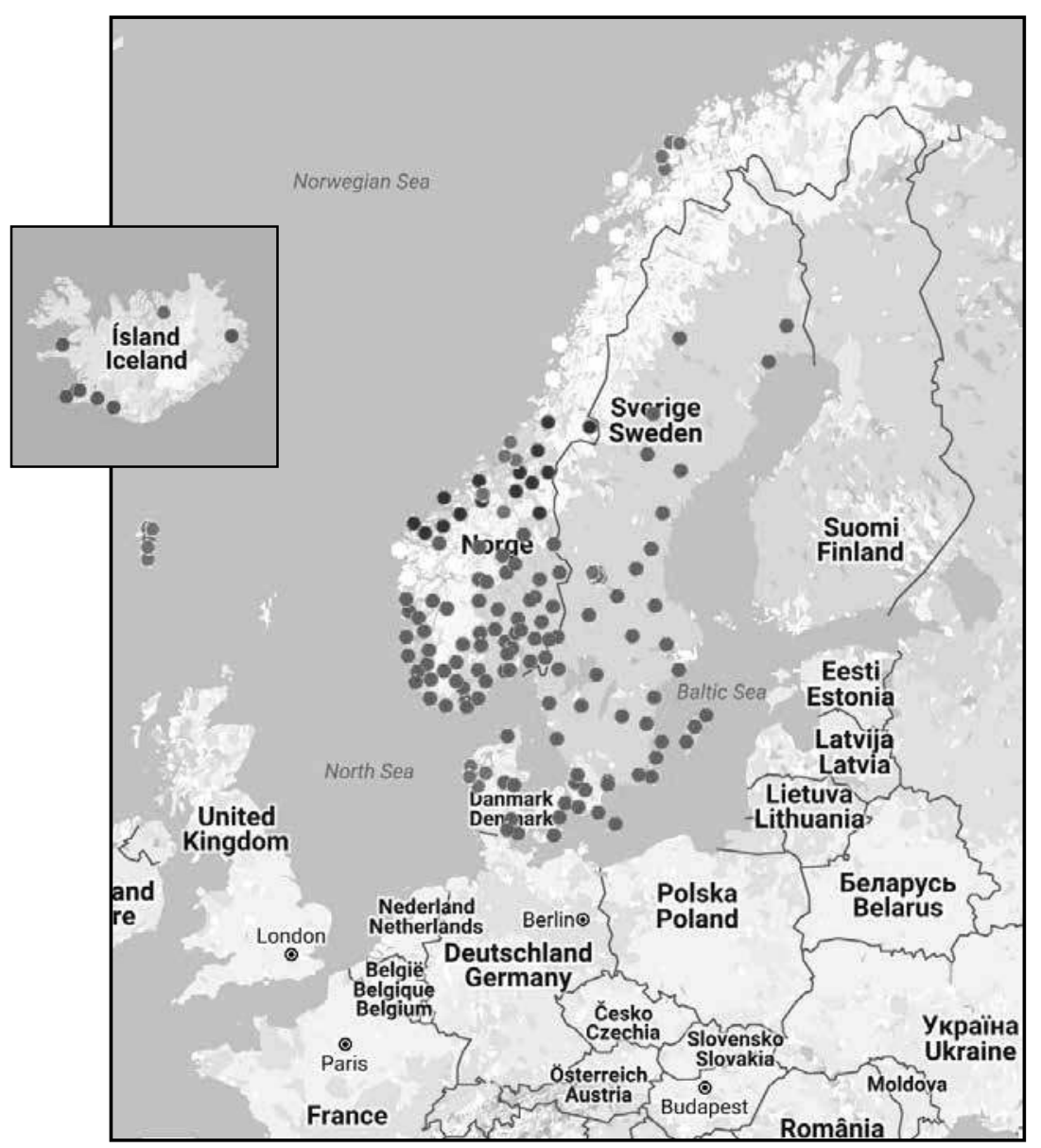

Carte 1. Indications géographiques des dialectes enregistrés, Målepunkt $i$ nordisk dialektkorpus (image tirée du site internet du corpus)

Extraits $d u$ corpus $^{8}$

Østfold (Norvège) - lieu : Fredrikstad

nikks \# alldri bodd uttafärr Freddriksta \#\# trivas har ja \# ja ha kke varrt no studdent heller da så kannsje ja hadde fLøtta litt menn de \# na ja ikke ha mått så \# vill ikke fLøtte haffra

" non \# je n’ai jamais habité en dehors de Fredrikstad \#\# je me plais ici \# je n’ai pas fait d'études sinon j'aurais peut-être déménagé un peu mais \# non je n'ai pas dû \# je ne veux pas déménager d'ici »

8 Le dièse «\# " indique une courte pause dans la production orale. "\#\# » renvoie à une pause légèrement plus longue. 
Senja (Norvège) - lieu : Botnhamn

du de bli jo vassjkeli spennans å se på denne gutstriken forr e \# husske du media korr damm krittiserte ledelsn i fjor forr damm ikkje tok hann ut te Oall? \# a vi vi henngte àss me i hylekairrpse tvarrt \#\# ja à kannsje de att damm så va fagfållk \# damm såg de ratte

« toi, ça sera super excitant de voir ce garçon car \# tu te souviens des médias comment ils critiquaient la direction l'année dernière, car ils l'avaient pas pris pour les Jeux olympiques ? \# et nous, nous poussions des cris avec les autres \#\# oui et peut-être qu'eux qui étaient des professionnels \# ils voyaient ce qui était juste »

Älvdalen (Suède) - lieu : Skolan

språkum lite jakt då : (ja) ja \# wen jäger denn pappa då? (näj an jäger fel brinder \# denn pappa då ?) ï ir fel brinder og byönn og \# fugler og \# liteï slaikt \# bruker du fy min og jågå?

"parlons un peu chasse alors : (oui) \# ton papa qu' est-ce qu'il chasse alors ? (ben il chasse plutôt l'élan \# et ton papa alors ?) c'est plutôt l'élan et l'ours et \# les oiseaux et \# comme ça \# tu as l'habitude d'aller chasser avec lui ?"

Vest-Jylland (Danemark) - lieu : Thorsminde

a har aldrig fäet lert at svømme det er også \# nej det er en fordel som fisker \# (ja de siger I) \# jamen som fisker der er det en fordel \# a er ligeglad for den dag den falder $i$ a vand du \# (ja ude à a hav)

« je n'ai jamais appris à nager, c'est aussi \# non c'est un avantage pour un pêcheur \# (aha tu le dis) \# oui pour un pêcheur c'est un avantage \# je suis autant heureux parce que le jour où je tombe dans l'eau tu \# (oui loin en mer) »

\section{Traitement automatique DeS DONNÉES}

Le dépouillement linguistique des données est principalement mené par le Tekstlaboratoriet de l'université d'Oslo. Une fois les enregistrements terminés, suivent les processus de transcription phonétique (a), de transcription orthographique (b), de lemmatisation (c), d'étiquetage morphosyntaxique (d) et d'enregistrement dans le moteur de recherche du corpus (e).

Tous les enregistrements norvégiens bénéficient d'une transcription phonétique et orthographique, ce qui n'est pas le cas pour toutes les données récoltées. Les points (c) et (d) sont différemment exécutés selon les langues nordiques. Les détails suivants, dont beaucoup sont pertinents pour l'ensemble du corpus, valent en premier lieu pour les dialectes norvégiens.

\section{Transcription phonétique}

La transcription consiste en la rédaction exacte de ce qui est prononcé, son après son. Elle suit les frontières de mots, et n'utilise que les lettres de l'alphabet latin (c'est-à-dire qu'elle n'utilise pas l'alphabet phonétique international, API). Elle 
doit aussi restituer la prononciation de la façon la plus fidèle possible. L'exemple fictif suivant (1) en est une illustration (voir les extraits de textes ci-dessus):

jai kåmm hit färr å hålle fåredrag åmm nåssjke dialekkter \# till onnge fåLLk jeg kom hit for å holde foredrag om norke dialekter til unge folk

« je suis venue ici pour faire un exposé sur les dialectes norvégiens aux jeunes "

$<æ>$ sera utilisé si l'informateur prononce [æ]. Le son [o] est transcrit par <å $>$ (comme dans hålle [faire], färedrag [exposé], etc.), le son [u], par $<\mathrm{o}>$ (exemple : onnge [jeunes]). $<$ sj> transcrit le son [s] (nåssjke [norvégiens]), et $<\mathrm{kj}>$, le son [ç] (par exemple kjue pour le chiffre 20).

Le L majuscule correspond au " $\mathrm{L}$ gras ", à savoir le flap rétroflexe [r] que l'on retrouve dans de nombreux dialectes norvégiens.

La variation entre le skarre-r ( $\mathrm{r}$ grasseyé) et le $\mathrm{r}$ roulé n'est pas marquée dans la transcription. La rétroflexion produite dans les combinaisons $r+n, r+t$ et $r+d$ n'est pas rendue visible, au même titre que les alvéolaires palatalisées (ll, nn, dd, tt $\rightarrow$ $\kappa, \mathrm{n}, \mathrm{J}, \mathrm{c})$.

Il est possible, si l'utilisateur s'intéresse à la prononciation, d'avoir recours aux fichiers audio, une fois que le mot ou l'expression a été repéré(e) dans le corpus?

La quantité phonique est signifiée par le redoublement de la consonne qui suit la voyelle courte : [VK:] $\rightarrow$ 'VKK' (alors que [V:K] $\rightarrow$ 'VK'). Dans l'exemple ci-dessus, kåmm, fårr, nåssjke, till, dialekkter, onnge, få LLk, le redoublement consonantique marque la durée de la voyelle qui le précède.

Ce procédé transcriptoire permet de lire aisément la prononciation des mots et des expressions, à l'exception des éléments marqués ci-dessus. Aussi, la transcription laisse deviner l'accent de mot dans les mots d'emprunts : gitar / gittar - garantert / garrantert.

Les voyelles courtes dans les suffixes flexionnels et dérivationnels ne sont pas indiquées par le redoublement de la consonne, car elles sont toujours non accentuées : -en et -dåm sont transcrits comme tels dans båten et barndåm (non pas båtenn og barndåmm).

La ponctuation est limitée : les majuscules sont utilisées pour les noms. Les points et les virgules ne sont pas utilisés. Le point d'interrogation marque l'intonation interrogative du locuteur. Le signe \# marque les pauses dans le discours. Les détails de la transcription sont accessibles dans le guide d'utilisation du Tekstlaboratoriet dans le lien indiqué en note de bas de page ${ }^{10}$.

9 Pour une description fondamentale du « L gras », des rétroflexes et de la palatalisation, etc. dans les dialectes norvégiens, voir par exemple Brit Mæhlum, Unn Røyneland, Det norske dialektlandskapet. Innforing i studiet av dialekter, Oslo, Cappelen Damm akademisk, 2012.

10 Les détails des conventions de transcription peuvent être consultés dans le manuel de transcription de Tekstlaboratoriet (www.tekstlab.uio.no/nota/ scandiasyn /Transkripsjonsrettleiing\%20for\%20 ScanDiaSyn.pdf). 
Il faut rappeler la difficulté liée à la transcription de discours spontanés, particulièrement quand il s'agit de personnes qui se connaissent, comme c'est le cas des données récoltées ici. La qualité du son, le débit du discours et le degré de recoupement des répliques entraînent des conséquences sur la précision de la transcription. Plusieurs dialectes peuvent, de surcroît, comprendre des phonèmes qui se placent entre deux réalisations orales de l'orthographe du bokmål, ce qui rend difficile le choix de la bonne lettre pour représenter ce qui est dit. Cela concerne entre autres le Vest-Telemark, où les participants plus âgés ont une voyelle courte qui se situe entre le [ø] et le [o] (par exemple, à Vinje). Les enregistrements audio doivent ainsi être utilisés si l'on souhaite contrôler les transcriptions.

\section{Transcription orthographique}

La transcription phonétique est faite mot pour mot suivant la norme linguistique écrite du bokmål, afin de simplifier l'exploitation du corpus et la recherche des variantes prononciatives d'un même mot. L'exemple ci-dessus : Jai kåmm hit fär à hålle färedrag åmm nåssjke dialekkter \# till onnge fåLLk sera donc traduit comme suit : jeg kom hit for à holde foredrag om norske dialekter \# til unge folk.

La translittération du stade phonétique au stade orthographique est effectuée de façon semi-automatique par un translittérateur spécialisé dans le traitement des données linguistiques, entraîné aux dialectes, qui propose tout d'abord une traduction automatique relue et corrigée par un assistant de transcription. Alors que l'objectif de la transcription phonétique est de reproduire le plus fidèlement possible la langue parlée, et de transcrire autant que faire se peut la variation, la transcription translittérale et orthographique vise à simplifier la recherche dans le corpus. La norme du bokmål est par conséquent strictement utilisée. Ainsi, les formes pronominales et les petits mots grammaticaux peuvent être traduits par des formes normées qui peuvent se distinguer fortement de la langue parlée. Cela facilite la recherche des différentes formes dialectales puisqu'un mot en bokmål entré dans le moteur de recherche renverra ainsi à toutes les variations parlées dans le corpus, y compris les formes similaires qui ne sont pas normées à l'écrit. Les exemples suivants illustrent les résultats obtenus lors d'une recherche par mots « normés ».
a. 'kånn' $\rightarrow$ oss
(pronom, $1^{\text {re }}$ personne, pluriel, forme objet) 'nous'
b. 'våssj' $\rightarrow$ oss
(pronom, $1^{\text {re }}$ personne, pluriel, forme objet) 'nous'
c. 'høtt' $\rightarrow$ hva (pronom interrogatif)
d. 'ka' $\rightarrow$ hva (interrogatif)
e. 'tå $\rightarrow a v \quad$ (préposition)
f. 'ta' $\rightarrow a v$ (préposition) 'quoi' 'quoi' 'de' 'de'

L'utilisateur intéressé par la morphosyntaxe doit savoir que les formes objet et sujet du pronom sont transcrites selon la norme écrite du bokmål. Le sujet 
formel der ([dårr], [dar] etc.), fréquent dans beaucoup de dialectes du Sud-Ouest norvégien, est transcrit par $<\operatorname{det}>^{11}$.

je ha prøvvd à kjøpe Metallika-sed'n dar nå fire gannger menn denn har dømm ikke (Aremark)

jeg har prøvd å kjøpe Metallica-cd-en der nå fire ganger men den har de ikke

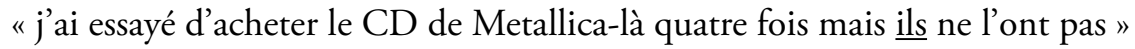
fårr dårr va enn a onngane så ikkje likkte \# tanngkmellkå (Gjesdal) for det var en av ungene som ikke likte \# tankmelka "... car il y avait un des jeunes qui n’aimait pas le lait cru "

La transcription et la translittération sont faites mot à mot, de sorte que les transcriptions phonétique et orthographique puissent être mises en relation.

jai kåmm hit fårr å hålle färedrag åmm nåssjke dialekkter \# färr onnge fåLLk jeg kom hit for å holdeforedrag om norske dialekter \# for unge folk " je suis venue ici pour faire un exposé sur les dialectes norvégiens aux jeunes "

Outre l'intérêt pour la linguistique informatique, une telle approche est essentielle pour les recherches menées sur la variation syntaxique. Par exemple, les dialectes où le verbe apparaît en troisième position (V3) dans les questions introduites par les pronoms interrogatifs commençant par $k v$ - (kva, kvifor, kven ${ }^{12}$, etc.), comme dans l'exemple suivant, et les dialectes où l'on observe la règle du verbe en deuxième position sont transcrits selon l'ordre des mots utilisés à l'oral.

$k a d u$ lik å jør på fritia? (Ballangen)

hva du liker å gjøre på fritida?

" qu'est-ce que tu aimes faire pendant ton temps libre?"

ko lika ru à jera i fritie ra? (Ål)

hva liker du à gjøre i fritida da?

"qu'aimes-tu faire pendant ton temps libre?"

La traduction mot à mot en une langue écrite normée exige de diviser des formes enclitiques dans la transcription phonétique, comme dans les exemples suivants :
a. [hakke] $\rightarrow$ 'ha kke' $\rightarrow$ har ikke
(n’a pas)
b. [bønnte] $\rightarrow$ 'børr nte' $\rightarrow$ bør ikke (n'habite pas)
c. [skaru] $\rightarrow$ 'ska ru' $\rightarrow$ skal du
(dois-tu)

11 Pour des informations supplémentaires, voir le manuel de translittération des dialectes de Tekstlaboratoriet (www.tekstlab.uio. no/nota/scandiasyn/oversetter-veiledning.pdf).

12 Quoi/que, pourquoi, qui. 
Quand deux types de transcription coexistent, elles sont mises en parallèle et rattachées aux enregistrements audio sur lesquelles elles sont fondées. La recherche peut simultanément porter sur deux transcriptions, de manière à comparer deux variantes. Il est également possible de chercher des variantes prononciatives d'un mot dans différents lieux géographiques. Dans tous les cas, les transcriptions peuvent être lues en même temps qu'il est possible de les écouter.

\section{Extrait du corpus, Alvdal, Norvège :}

A : ja na føLLkevånngna nå ha ru denn ell ?

ja denne folkevogna nå har du den eller?

"donc cette Volkswagen, tu l'as maintenant, n'est-ce pas?"

B : ja ra e har a ho står borrtpå låven hænn Hellge skulle fått inn ja da jeg har henne hun stär bortpå låven han Helge skulle fätt inn "Oui, je l'ai, elle est dans la grange, Helge devait lui installer "

ny mottor i a fårr re va denn såmm bynnt å...

ny motor $i$ henne for det va den som begynte $a$...

"un nouveau moteur car c'était lui qui commençait à..."

A : hanngLe?

«brouter?»

B : hanngLe e hørte re sjø nå je va på mølltetur fårr sisste sømmærn hangle jeg hørte det sjø når jeg var på multetur for siste sommeren "brouter, je l'ai entendu sjø quand j'allais ramasser des baies polaires l'été dernier »

\section{Lemmatisation}

Après transcription et translittération, les données sont lemmatisées et étiquetées sur le plan morphosyntaxique. La lemmatisation implique d'ajouter des informations sur le mot d'entrée, auquel sont identifiées les formes flexionnelles. Cela rend possible une recherche par lemme qui donne toutes les variantes du mot, que cela soit toutes les formes temporelles du verbe ou les formes plurielles ou singulières, indéfinies ou définies du nom.

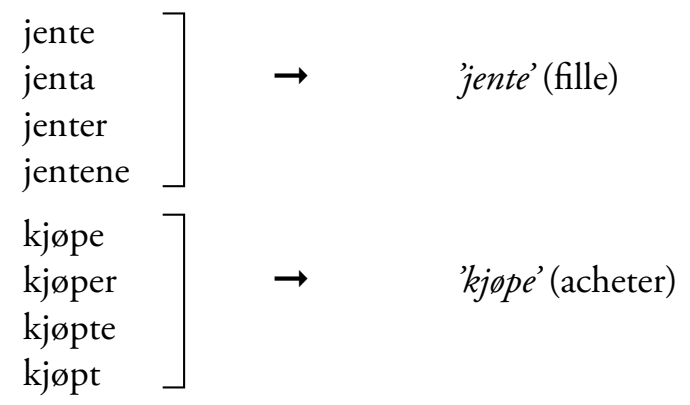

Ce processus est effectué de manière automatique à l'aide d'un étiqueteur de langue orale, à partir de la transcription orthographique en bokmål. 


\section{Étiquetage}

L'étiquetage est un processus en linguistique informatique qui ajoute des informations grammatico-sémantiques à un mot qui entre dans le corpus. Dans le Nordisk dialektkorpus, les transcriptions sont annotées par lemme, par catégorie grammaticale et par flexion.

À l'écoute et à la lecture, l'utilisateur peut, outre le lemme, identifier à quelle catégorie grammaticale ou dans quelle forme flexionnelle se placent tous les mots de l'énoncé. La recherche peut également être limitée à quelques catégories grammaticales et/ou à quelques catégories flexionnelles. La nomenclature suit en grande partie Norsk referansegrammatik $k^{13}$.

Catégorie grammaticale : verbe, nom, adjectif, pronom, déterminant, préposition, adverbe, conjonction, subjonction (conjonction de subordination), interjection.

Flexion : temps, nombre, genre, définitude, personne, comparaison, cas.

Sous-catégories : déterminants : quantificateur, adjectif possessif, démonstratif; noms : nom commun, nom propre.

Les exemples suivants illustrent les catégories identifiées (les descriptions renvoient ici au terme indiqué en italique) :

\begin{tabular}{|l|l|l|l|}
\hline jenta & «jente » & 'fille' & nom, féminin, singulier, forme définie \\
\hline kjøpte & «kjøpe » & 'acheter' & verbe, prétérit \\
\hline henne & « hun » & 'elle' & $\begin{array}{l}\text { pronom personnel, 3 personnes, féminin, } \\
\text { singulier, accusatif }\end{array}$ \\
\hline mitt & «min » & 'mon' & déterminant, possessif, neutre, singulier \\
\hline fint & «fin» & 'beau' & adjectif, neutre, singulier, indéfini, positif \\
\hline på & «på » & 'sur' & préposition \\
\hline
\end{tabular}

Exemple du corpus, Alvdal, Norvège:

\begin{tabular}{|l|l|ll|l|l|}
\hline Phonétique & Orthographique & \multicolumn{2}{|c|}{ Lemme } & Catégorie & \multicolumn{1}{c|}{$\begin{array}{c}\text { Forme } \\
\text { flexionnelle }\end{array}$} \\
\hline e & jeg & jeg 'je' & pronom & $1^{\text {re }}$ p. sg. nom. \\
\hline har & har & ha 'a' & verbe & présent \\
\hline a & henne & hun 'la' & pronom & $3^{\text {e }}$ p. sg. acc. \\
\hline ho & hun & hun 'elle' & pronom & $3^{\text {e p. sg. nom. }}$ \\
\hline står & står & stå 'être' & verbe & présent \\
\hline borrtpå & bortpå & bortpå 'sur' & préposition & - \\
\hline låven & låven & låve 'grange' & substantif & masc. sg. def. \\
\hline
\end{tabular}

13 Jan T. Faarlund, Svein Lie, Kjell Vannebo, Norsk referansegrammatikk, Oslo, Universitetsforlaget, 1997. 
L'étiquetage se fait de manière automatique par un logiciel d'étiquetage oral créé lors du développement du corpus NoTa-Oslo" . Il reprend l'outil "OsloBergen » développé pour la langue écrite.

Létiqueteur de la langue orale a été une première fois testé sur un enregistrement du dialecte d'Oslo transcrit en bokmål, identifié manuellement par un assistant de transcription.

Le processus d'étiquetage est réalisé de telle sorte qu'une partie importante du corpus est annotée à la main, pour que cela soit utilisé comme mesure dans le processus statistique informatisé.

Pour chaque analyse de mot, l'étiqueteur automatisé prend en compte les deux mots précédents (lemme, catégorie et forme flexionnelle), les étiquettes possibles du mot, les trois dernières lettres du mot, etc.

Les résultats du test montrent que le logiciel a un niveau de précision d'environ 96 \%. Les mots qui entraînent le plus d'erreurs sont de petits mots grammaticaux, comme så det et $f o r^{15}$. Så pose des difficultés particulières, car il a quatre interprétations possibles (prétérit du verbe $s e$ [voir], adverbe, conjonction ou subjonction $\left.{ }^{16}\right)$ :

vi bodde hos han i tre måneder så bodde vi sju måneder $i$ eget hus (adverbe) " on a habité chez lui pendant trois mois, donc on a habité sept mois dans notre maison"

men han e viste seg à vere litt gal så vi måtte flytte fra han (conjonction) " mais il s'est révélé être un peu fou et alors nous avons dû le quitter " vi må synge den oftere, så vi ikke glemmer den. (subjonction) « il faut qu'on la chante plus souvent de sorte que nous ne l'oubliions pas »

Selon Norsk referansegrammatikk ${ }^{17}$, la conjonction så introduit une proposition principale. Le contenu exprime souvent la conséquence. La subjonction så introduit une subordonnée et exprime un but ${ }^{18}$. L'adverbe så peut avoir plusieurs fonctions, comme lier contextuellement des phrases entre elles. L'utilisation de ces catégories grammaticales dans tous les exemples avec så peut s'avérer problématique. Par

14 www.tekstlab.uio.no/nota/oslo/

15 Åshild Søfteland, Anders Nøklestad, « Manuell morfologisk tagging av NoTa-materialet med støtte fra en statistisk tagger ", in Språk i Oslo. Ny forskning omkring talespråk, K. Hagen et J. B. Johannessen (dir.), Oslo, Novus, 2008, p. 228.

16 Ibid., p. 230.

17 Jan Terje Faarlund et al., op. cit.

18 Åshild Søfteland, Anders Nøklestad, op. cit., p. 230. 
exemple, så peut être syntaxiquement utilisé comme conjonction sans qu'il y ait sémantiquement une conséquence ${ }^{19}$, comme dans l'exemple suivant :

det er Oslo altså Skullerud så \# jeg har bodd her $i$ åtte år nå (conjonction ?)20

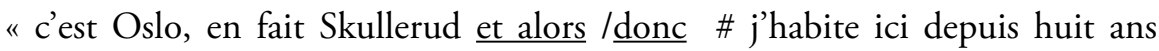
maintenant"

L'analyse de nombreux cas révèle un modèle que l'on peut suivre en pratique : så comme subjonction est peu fréquent ; så est le plus souvent utilisé comme conjonction quand il est suivi d'un syntagme nominal ; så est adverbe quand il est suivi par un verbe conjugué, sauf quand il y a ellipse du sujet. L'exemple suivant comprend un sujet jeg sous-entendu (ibid. $)^{21}$ :

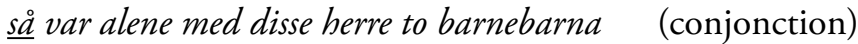

"... et alors étais avec les deux petits enfants de ces messieurs »

\section{Chercher dans le corpus}

Une fois les processus ci-dessous menés à bien, la recherche est possible dans une interface adaptée, et les transcriptions (comprenant la lemmatisation et l'étiquetage morphosyntaxique) sont liées aux fichiers audio et éventuellement vidéo.

\section{RECHERCHER LA VARIATION DIALECTALE DANS LE CORPUS}

L'accès au Nordisk dialektkorpus se fait par des sites internet spécifiques ${ }^{22}$. Nous détaillerons ici les modalités de recherches pour trouver les données orales ou dialectales, que ce soit par une recherche simple ou avancée. Dans la bibliographie, on trouvera des liens vers des tutoriels qui illustrent les types de recherches mentionnées ci-dessous.

19 Ibid., p. 231.

20 Un relecteur propose que cette occurrence de så doit plutôt être catégorisée comme une particule discursive. Nous sommes tout à fait d'accord avec cette idée, mais dans le travail actuel nous avons dû appliquer de près les catégories établies dans Norsk referansegrammatikk et l'étiqueteur Oslo-Bergen afin de pouvoir au mieux effectuer des calculs statistiques. Nous avons alors noté comme problématiques des cas comme celui-ci.

21 Voir Anders Nøklestad, Åshild Søfteland, «Tagging a Norwegian Speech Corpus », in NODALIDA 2007 Conference Proceedings, J. Nivre, H.-J. Kaalep, K. Muishnek et M. Koit (dir.), 2007, p. 245-248. (http://dspace.ut.ee/bitstream/handle/10062/2582/stud-Nklestad-20.pdf). Voir également Åshild Søfteland, Anders Nøklestad, op. cit. pour une introduction sommaire à l'étiqueteur de langue orale. Pour des informations plus détaillées sur les critères de l'analyse grammaticale appliqués dans l'étiquetage fait manuellement, entre autres quand il s'agit des cas clairs d'ambiguïté, voir le manuel de Tekstlaboratoriet (www.tekstlab.uio.no/nota/oslo/Taggeveiledning2.pdf). Pour un survol général de l'annotation dans le Nordisk dialektkorpus, voir Janne B. Johannessen, "Annotations in the Nordic Dialect Corpus ", in Handbook of Linguistic Annotation, N. Ide et J. Pustejovsky (dir.), New York, Springer, 2017, p. 130-132.

22 www.tekstlab.uio.no/nota/scandiasyn, ou https://tekstlab.uio.no/glossa2/ndc. Il est possible de s'y connecter via Feide ou eduGain. 


\section{Recherche simple}

\section{Thème: pronoms interrogatifs}

Objectif: trouver les variations dialectales norvégiennes du pronom interrogatif hva (quoi/que)

Méthode : lancer une recherche avec hva et délimiter la région à "Norway ".

Si l'on veut seulement trouver des prononciations spécifiques, il est possible de les chercher directement. Il faut, pour ce faire, entrer la forme spécifique, comme høtt ou $k a$, et chercher dans la transcription phonétique en sélectionnant "phonetic".

Thème : sujet formel

Objectif: étudier l'alternance entre det ou der comme sujet formel dans différentes constructions en danois.

Méthode : chercher les formes det et der et choisir « Denmark ».

Pour une double recherche, sélectionner « or...» (ou) sous la barre de recherche. Les résultats s'affichent d'abord selon le lieu de l'enregistrement, mais ils peuvent également être présentés de façon alphabétique en fonction des mots choisis.

\section{Recherche avancée}

\section{Thème : flexion du nom}

Objectif: trouver les variations flexionnelles dialectales des noms masculin et féminin en norvégien.

Méthode: choisir " recherche avancée " (avansert søk) puis " Norway ".

Plusieurs façons de faire sont possibles :

1) chercher par exemple les mots gutt (garçon) et jente (fille) (ou tout autre nom courant) et choisir " lemma" (lemme) pour trouver toutes les variantes flexionnelles. Il faut éventuellement délimiter en sélectionnant uniquement " pluriel » et/ou seulement "forme définie ». Pour cela, il faut ouvrir le menu déroulant et choisir « noun ", " plural» et/ou « definite».

2) choisir un lieu dans l'onglet "Region », par exemple " Østlandet ", chercher tous les noms à la forme définie plurielle en sélectionnant " noun », " plural » et "definite ». Il est possible de limiter davantage les résultats en sélectionnant un critère supplémentaire, en ajoutant une recherche pour les mots masculins, signalisée par «masculine». D’autres combinaisons sont possibles.

Thème: position du verbe

Objectif : trouver les frontières des dialectes qui utilisent le verbe en troisième position dans les questions introduites par un pronom interrogatif en -kv(voir supra). 
Méthode: il y a plusieurs approches possibles, mais toutes impliquent une recherche d'expressions comprenant plusieurs mots, rendue possible en sélectionnant " + " dans le moteur de recherche.

- Choisir des combinaisons de mots définis. Par exemple, la forme orthographique hva, combinée avec le pronom $d u$, la catégorie " verb» et " present ». Il faut ensuite écarter les exemples non pertinents pour s'assurer que l'on n'analyse que les questions commençant par un pronom interrogatif en $k v$-.

- Lancer une recherche plus large en choisissant des mots qui commencent par $k v$ - dans la première ligne, puis "pronomen " (pronom) dans la deuxième et " finitte verb " (verbe conjugué) dans la troisième. Pour chercher un pronom, il faut choisir « pronoun » dans le menu déroulant. Pour chercher des verbes conjugués, il faut sélectionner " verb », puis " present » et " past ». On peut indiquer hv dans le moteur de recherche pour trouver un mot qui commence par $k v$-. De la sorte, tous les mots qui commencent par $h v$-dans la transcription orthographique apparaîtront. Cet exemple, théorique, donnerait de très nombreux résultats. Pour faciliter la recherche, elle pourrait être limitée à une région à la fois pour réduire le nombre de résultats.

Les données peuvent être traitées de plusieurs façons. Le plus important est d'avoir toujours accès à l'enregistrement audio. Parfois, un enregistrement vidéo est également accessible. L'utilisateur peut également télécharger tous les résultats dans un fichier (de type Excel, ou autre). Les résultats peuvent également être visualisés sur la carte des pays nordiques, et l'on peut les trier avec des codes couleur selon les prononciations. L'interface permet également de voir les ondes sonores de l'enregistrement, sous la forme de graphiques (spectrogrammes). Les textes comprennent un lien hypertexte vers Google Translate qui permet de traduire grossièrement la transcription orthographique de la langue source en anglais.

\section{UTILISATION DES DONNÉES DANS LA RECHERCHE ET L'ENSEIGNEMENT : QUELQUES EXEMPLES}

Nous proposons quatre exemples réels d'exploitation des données issues du Nordisk dialektkorpus. Ces exemples ont été utilisés dans une thèse de doctorat, un article scientifique, un contenu de cours et un projet de recherche dans sa phase initiale. 


\section{Det comme sujet dans le parler spontané en norvégien}

Le premier exemple est tiré de la thèse de doctorat de l'auteure ${ }^{23}$, qui comprend, entre autres, des analyses quantitatives et qualitatives du mot det comme sujet. La recherche se fonde sur l'analyse de quatre localités (Alvdal, Senja, Suldal, Oslo ${ }^{24}$ ), chacune étant représentée par quatre informateurs. L'étude porte sur toutes les phrases où det apparaît comme sujet, dans huit conversations, et leur transcriptions phonétiques quand celles-ci sont accessibles. Tous les detsujets ont été ensuite catégorisés en sous-types, selon qu'ils soient référentiels ou formels, et selon ce à quoi ils font référence ou dans quel type de construction impersonnelle ils s'inscrivent :

det référentiel $=>$ à quoi renvoie det ?

det formel $=>$ de quelle sorte de construction impersonnelle s'agit-il ?

Det peut renvoyer à des mots simples, des syntagmes, des propositions ou des morceaux de proposition et des paragraphes entiers. En pratique, il est rarement évident de savoir ce à quoi det renvoie, comme dans l'exemple $\mathrm{B}$ ci-dessous ${ }^{25}$ où det peut renvoyer à la phrase précédente ou au passage $\mathrm{A}$ dans son intégralité :

A : Og så stod det masse gamle tyskerbrakker igjen der. Noen var revet og noen bodde det enten sigøynere eller et eller annet sånn e $m$ merkelig folk i. [...] Og ee men de stedene hvor b-selve brakka var borte, når de u-em grunnmuren sto igjen, der lekte vi inni grunnmuren. (Oslo)

B : Det var spennende selvfølgelig. (Oslo)

$\mathrm{A}:$ : il restait plein de baraques d'Allemands là. Certaines ont été démolies et d'autres étaient habitées, par des gitans ou d'autres gens bizarres. [...] Mais les endroits où les baraques avaient disparu, s'il restait les fondations, alors on jouait dans les fondations" (Oslo)

$\mathrm{B}:$ «ćétait passionnant, bien sûr »(Oslo)

Det revient également fréquemment comme sujet formel en norvégien, par exemple dans les phrases présentatives, les structures clivées, le passif impersonnel et dans les constructions avec les verbes météorologiques. En pratique, il peut toutefois être difficile de déterminer la nature du det, comme dans l'exemple ci-dessous $^{26}: s^{\prime}$ agit-il d'une construction clivée (C'est $[\mathrm{X}]$ qui $\mathrm{Y}$ ) ou d'une simple construction présentative (C'est $\underline{\mathrm{X}}$ ) avec une phrase relative restrictive incluse dans le sujet réel ?

23 Åshild Søfteland, Utbrytingskonstruksjonen i norsk spontantale (La construction clivée en norvégien oral spontané), thèse de doctorat, Université d'Oslo, 2014 (https://www.duo.uio.no/handle/10852/39403).

24 Le matériau d'Oslo vient du corpus NoTa.

25 Åshild Søfteland, op. cit., p. 158.

26 Ibid., p. 152. 
Sånne amerikanarar som sender inn videoar som dei har filma sjolv, der er det alltid [noko sånt]_som står og dansar på bordet eller noko bryllupsgreier, alltid noko. (Suldal)

" ce genre d'Américains qui nous envoient des vidéos qu'ils ont eux-mêmes filmées, il y a toujours quelqu'un qui est en train de danser sur la table ou des trucs de mariage, toujours quelque chose » (Suldal)

Dans ce contexte, il est difficile de déterminer s'il s'agit d'un det référentiel ou non référentiel et la question est relativement peu traitée dans la littérature scientifique $^{27}$. Nous constatons ${ }^{28}$ : "Environ un tiers de tous les sujets en $\operatorname{det}$ dans les données [est analysé comme référentiel], mais les statistiques cachent beaucoup de cas discutables. " Les exemples suivants illustrent cette ambiguïté29 :

(Contexte : l'informateur doit vendre sa voiture mais en exige au moins 135000 couronnes)

Men det er mange som synst det er dyrt. (Alvdal)

"mais il y en a beaucoup qui pensent que c'est cher"

$\rightarrow$ Det er mange som synst det er dyrt 135.000 .

"beaucoup pensent que 135000 couronnes, c'est cher "

$\rightarrow$ (Det er mange som synst) det ${ }_{i}$ er dyrt.

" beaucoup pensent que 135000 couronnes est cher»

Det peut être soit sujet formel dans une construction impersonnelle avec une phrase prépositionnelle sous-entendue, soit renvoyer au montant de $135000 \mathrm{cou}-$ ronnes ${ }^{30}$. Les deux interprétations sont possibles dans le contexte et les données récoltées montrent de nombreux cas similaires.

$\mathrm{Si}$ l'on compare les analyses issues de notre thèse ${ }^{31}$ avec les recherches précédentes, il semble évident que les données orales spontanées du Nordisk dialektkorpus ouvrent beaucoup de perspectives ; beaucoup de définitions traditionnelles doivent être reformulées pour illustrer les formes qui apparaissent. L'utilisation du corpus permet de développer les connaissances sur les références anaphoriques, sur la catégorisation des constructions impersonnelles et sur la structure informationnelle à la fois en norvégien et plus généralement.

27 Mais voir entre autres Elisabeth Engdahl, "Relative Clause Extractions in Context ", Working Papers in Scandinavian Syntax, 60, 1997, p. 59-86 ; Marketta Sundman, Existentialkonstruktionen i svenskan, Åbo, Åbo Akademi, 1980 ; Kari Kinn, «Referential vs. non-referential null subjects in Middle Norwegian ", Nordic Journal of Linguistics, 39(3), 2016, p. 277-310 ; Susannah Kirby, Misha Becker, "Which it is it? The Aqcuisition of Referential and Expletive it ", Journal of Child Language, 34, 2007, p. 571-599.

28 Åshild Søfteland, op. cit., p. 158.

29 Ibid.

30 Åshild Søfteland, op. cit., p. 159.

31 Ibid. 


\section{Sjø en trøndersk (dialecte de Trondheim)}

Borthen et Søfteland ${ }^{32}$ ont mené une recherche sur la particule sjø en trøndersk, dans le cadre d'une analyse des particules discursives dans les dialectes norvégiens. Sjø provient probablement de l'expression ser $d u$ (vois-tu), équivalente au you see anglais. Il s'agit d'une particule discursive connue sur laquelle toutefois peu de recherches ont été menées. Une recherche simple du terme sjø dans le corpus donne plus de 400 résultats. Relativement peu de réponses renvoyaient à sjø dans le sens de " mer » ou « lac ». En pratique, il n'y avait aucune ambiguïté entre les deux lexèmes, ce qui a facilité l'identification des objets d'analyse :

A : Kva gjorde du etter det da, var det tok du noko arbeid? (chercheur) " qu'as tu fait après ça, t'as pris un travail ?"

B : Nei eg gjorde ikkje det til à begynne med sjø, for eg var heime med ungane. " non j'ai pas fait ça pour commencer sjø, car j'étais à la maison avec les enfants"

Det var ikkje noko barnehage eller noko sånn åt ungane da sjø. (Røros)

"il n'y avait pas de crèches ou ce genre de choses pour les enfants à cette époque $s j ø "$ (Røros)

La transcription du discours oral dans une orthographe standardisée est ici d'un grand avantage, car le terme sjø n'existe pas dans le dictionnaire officiel du bokmål (Bokmålsordboka). Les différentes prononciations comme [sje], [sju] et [sji] ont toutes été orthographiées $<$ sjø $\varnothing$. Ainsi, elles peuvent être repérées par une recherche simple. Cela a permis la publication d'un article sur la répartition géographique et les modes d'utilisation de sjø, jusqu'alors inconnus dans la recherche, offrant des analyses sémantico-pragmatiques avancées.

Outre une connaissance approfondie du norvégien parlé, le corpus, et les analyses qu'il autorise, peuvent contribuer au développement de la théorisation internationale sur le même sujet.

\section{Comparaisons des parlers générationnels à Alvdal}

Le Nordisk dialektkorpus peut aussi devenir une ressource très utile pour l'enseignement en dialectologie, par exemple en cursus de norvégien ou d'études nordiques dans l'enseignement supérieur, par l'écoute de dialectes des pays nordiques ou par l'analyse sociolinguistique ou grammaticale d'extraits plus longs. Les données norvégiennes du corpus comprennent habituellement quatre informants par localité : une jeune femme, un jeune homme, une femme plus âgée et un homme plus âgé. Cela donne la possibilité d'étudier les changements linguistiques. Quels

32 Kaja Borthen, Åshild Søfteland, « Æ e trønder, $x$, sjø! », in Den pragmatiske partikkelen sjø i midtnorske dialektar, à paraître. 
traits dialectaux semblent disparaître chez les plus jeunes ? Un bon point de départ pourrait être l'étude de la transcription des dialogues de la localité d'Alvdal et la comparaison de la langue des informateurs plus jeunes et plus âgés à partir d'une liste des caractéristiques emblématiques. Les étudiants pourraient trouver un modèle comme suit :

\begin{tabular}{|l|c|c|c|}
\hline $\begin{array}{l}\text { Marqueur } \\
\text { linguistique } \\
\text { (sélection) }\end{array}$ & Alvdal (âgés) & Alvdal (jeunes) & \\
\hline $\begin{array}{l}\text { Pronom } 1^{\text {re }} \text { personne } \\
\text { du singulier }\end{array}$ & ai ou je & $j e$ ou jai & "je " \\
\hline Négation & itte & $i k k e$ & "ne pas » \\
\hline Suffixe infinitival & a komma & a komme & "venir » \\
\hline $\begin{array}{l}\text { Ouverture de } \\
\text { voyelles }\end{array}$ & sekkert, tell & sikkert, till & " sûr, à » \\
\hline Datif & ei ta jenntom hass & ei ta jennt’ne hass & " une de ses filles » \\
\hline
\end{tabular}

Les étudiants peuvent étudier l'analyse grammaticale à des niveaux différents, tout en réfléchissant sur les changements linguistiques en pratique et les paramètres sociolinguistiques potentiellement à l'œuvre.

\section{Les traits traditionnels dans le dialecte de l'Østfold}

L'Institut supérieur d'Østfold (Høgskolen i Østfold) a lancé un projet sociolinguistique de grande ampleur sur la pratique linguistique dans cette région. Le point d'entrée peut être d'étudier les traits dialectaux traditionnels qui sont conservés dans les enregistrements réalisés dans l'Østfold, en lien avec le développement du Nordisk dialektkorpus.

Il y a trois localités du comté enregistrées dans le corpus, avec quatre informateurs par localité, autant de jeunes que de personnes plus âgées. Plusieurs recherches ont été lancées dans la région à partir des marqueurs linguistiques suivants :

\begin{tabular}{|l|c|c|c|}
\hline $\begin{array}{l}\text { Marqueur } \\
\text { linguistique }\end{array}$ & $\begin{array}{c}\text { Forme } \\
\text { traditionnelle }\end{array}$ & Forme récente & \\
\hline $\begin{array}{l}\text { Pronom, } 3^{\mathrm{e}} \text { personne } \\
\text { du pluriel (1) }\end{array}$ & dømm-dømm & $\begin{array}{c}\text { di-di eller } \text { di- } \\
\text { demm }\end{array}$ & "ils, elles " \\
\hline Négation (2) & ennte & ikke & " ne pas » \\
\hline Suffixe infinitival (3) & à varra & å vere & " être " \\
\hline $\begin{array}{l}\text { Nom masculin au } \\
\text { pluriel (4) }\end{array}$ & båter, båtane & båter, båtne & "bateaux " \\
\hline $\begin{array}{l}\text { Accentuation de } \\
\text { mots d'emprunt (5) }\end{array}$ & stassjon & stasjon & "gare " \\
\hline
\end{tabular}


Pour trouver des informations sur les formes qui sont utilisées, la recherche est ici localisée dans la région de l'Østfold (area : Østfold).

Pronom (1) : Étant donné que toutes les formes pronominales ont été converties en bokmål normé, y compris morphologiquement, on peut rechercher la forme orthographique " de » et ainsi obtenir toutes les variantes orales du pronom personnel à la $3^{\mathrm{e}}$ personne du pluriel à fonction sujet. Dans le corpus d'Østfold, on observe que $85 \%$ des occurrences orthographiques de " de " renvoient aux prononciations se terminant avec $-m$, majoritairement dømm ou rømm, mais également demm et domm.

Négation (2) : Ici, on recherche facilement le mot orthographique <ikke>, ensuite trié par variante phonétique, par exemple ennte, ikke, ikk et kke. La forme ennte et le clitique nnte constituent environ $15 \%$ des variantes de la négation. On constate que différentes versions de itte sont légion à Rømskog dans la partie nord-est de la région d'Østfold.

Infinitif (3) : L'interface se prête bien à la recherche de "verbe » et "infinitif ", tout en limitant la recherche à ceux qui se terminent par $-a$ ' en transcription phonétique. Ensuite peuvent être supprimés les infinitifs monosyllabiques qui ne montrent pas la variation concernée, comme ha et $d r a^{33}$. De cette manière, on peut identifier toutes les occurrences des infinitifs disyllabiques sur $-a(\mathrm{~N}=$ 128), et ce résultat peut ensuite être mis en relation avec la recherche par mots spécifiques, comme toutes les variantes phonétiques de <være> (être; $\rightarrow$ autour de $40 \%$ sur $-a$ ).

Masculin pluriel (4) : Ici on peut commencer en recherchant en transcription phonétique tous les noms qui se terminent par - ar ou -ane. On obtient alors un certain nombre d'occurrences de -ane, la forme traditionnelle au masculin défini pluriel, et encore plus de -ar, le suffixe traditionnel de la forme indéfinie. Il convient cependant de comparer ces résultats avec une recherche des mots se terminant par-er et -ene afin d'obtenir des informations quantitatives utiles.

Accentuation sur la première syllabe (5) : Cette tâche est la plus compliquée des cinqs évoquées ici. Les mots d'emprunt ne sont pas spécifiquement annotés dans le corpus, mais on peut chercher sur « lemme», et ensuite " mot se terminant par-sjon " et " mot se terminant par-ere ", en supprimant les résultats non pertinents. De plus, on peut rechercher des mots spécifiques comme musikk, gitar et dialekt. Étant donné que nous annotons la durée de la voyelle ou de la consonne en syllabe accentuée, on peut souvent déduire où se trouve l'accent de mot grâce à la transcription. Voir stassjon vs. stasjon, studdere vs. studere og dialekt vs. dialekkt.

33 Pour une explication du concept de jamvekt (la prosodie des infinitifs disyllabiques), voir par exemple Brit Mæhlum, Unn Røyneland, op. cit. 
L'analyse de 130 mots de ce type, qui apparaissent une fois ou plus dans le corpus, nous informe qu'environ 60 \% d'entre eux sont accentués à la première syllabe.

Le calcul global ne permet pas de se prononcer de manière univoque sur les changements linguistiques dans l'Østfold, car l'on trouve d'importantes divergences au niveau individuel, ainsi qu'entre les différents lieux d'enregistrement. Cependant, il semblerait que les formes suivantes soient bien maintenues : la forme pronominale dømm, accentuation à la première syllabe (voir stassjon), et partiellement la flexion masculine du nom (par exemple gutter). Par contre, les infinitifs disyllabiques sur $-a$ (voir varra) et la négation ennte se préservent moins bien.

Encore d'autres ouvrages ${ }^{34}$ fournissent des exemples de l'exploitation du Nordisk dialektkorpus et de NoTa-Oslo. Ce dernier corpus suit les mêmes principes que le premier, mais n'applique que la transcription orthographique.

\section{ConClusion}

Le Nordisk dialektkorpus, englobant la totalité du Norden, est un outil de recherche fort utile, susceptible de nous apprendre beaucoup sur la grammaire, la pragmatique et la sémantique dans l'optique soit traditionnellement dialectologique, soit sociolinguistique, auxquelles s'ajoutent les enseignements de la prononciation et des stratégies communicatives. Cet article s'est donné comme objectif d'informer et de donner des illustrations de la situation d'enregistrement, la transcription, le traitement automatique des données et la recherche dans le corpus, en espérant qu'un nombre croissant de linguistes pourront se servir de ce corpus dans leurs recherches aussi bien que dans leur enseignement.

\section{Traduction de Sarah Harchaoui}

34 Janne B. Johannessen, Joel Priestley, Kristin Hagen, Tor A. Åfarli, Øystein A. Vangsnes, "The Nordic Dialect Corpus : an Advanced Research Tool », in NEALT Proceedings Series, vol. 4, K. Jokinen et E. Bick (dir.), 2009, p. 73-80 (http://omilia.uio.no/nealt) ; Kristin Hagen, Janne B. Johannessen, op. cit. ; Språk i Norge og nabolanda. Ny forskning om talespråk, J. B. Johannessen et K. Hagen (dir.), Oslo, Novus, 2014 ; revue Nordic Atlas of Language Structures (NALS), http://www.tekstlab.uio.no/nals\#/. 


\section{ANNEXe}

\section{Sitographie}

Nordisk dialektkorpus : http://tekstlab.uio.no/glossa/html/?corpus=scandiasyn

ScanDiaSyn (Syntaxe des dialectes nordiques) : http://websim.arkivert.uit.no/scandiasyn/ Corpus ScanDiaSyn : http://www.tekstlab.uio.no/scandiasyn/base

Corpus NoTa-Oslo : www.tekstlab.uio.no/nota/oslo/

Archive des dialectes (Målførearkivet) : www.uib.no/lle/73605/meir-om-målføresamlinga SweDia 2000 (Dialectes en Suède) : http://swedia.ling.gu.se/

Étiqueteur Oslo-Bergen : www.tekstlab.uio.no/obt-ny/

Revue NALS : http://www.tekstlab.uio.no/nals\#/

Laboratoire de textes : http://www.hf.uio.no/iln/om/organisasjon/tekstlab/

Manuel de transcription : www.tekstlab.uio.no/nota/ scandiasyn/Transkripsjonsrettleiing\%20for\%20 ScanDiaSyn.pdf

Manuel de translittération : www.tekstlab.uio.no/nota/scandiasyn/oversetter-veiledning. pdf

Manuel d'étiquetage : www.tekstlab.uio.no/nota/oslo/Taggeveiledning2.pdf

Information sur la collecte des données : www.tekstlab.uio.no/nota/scandiasyn/dialect_ data_collection.html

Site pour log-in direct à l'interface : https://tekstlab.uio.no/glossa2/ndc 